\title{
Transitional cell carcinoma of endometrium: a case report of rare pure form
}

\author{
Francesco Cuccia, MD!, Gianluca Mortellaro, MD², Antonio Spera, MD!, Giovanna Evangelista, MD², \\ Prof. Antonio Lo Casto, MD³, Giuseppe Ferrera, MD² \\ IRadiotherapy Unit, ARNAS-Civico, University of Palermo, ${ }^{2}$ Radiotherapy Unit ARNAS-Civico Palermo, ${ }^{3}$ Sezione di Scienze Radiologiche. \\ DIBIMED, Università di Palermo, Palermo, Italy
}

\begin{abstract}
Transitional cell carcinoma is a very rare histological subtype of endometrial cancer, with only 18 cases described in literature. Ovary represents the most common site where it accounts for $2 \%$ of all ovarian cancers. Due to the rarity of this disease, current management is controversial and includes various approaches, mostly described in the adjuvant setting, both radiotherapy and chemotherapy alone or in combination. Here, we report a case of a 65-year-old patient who underwent adjuvant vaginal brachytherapy for an endometrial transitional cell carcinoma. The patient was treated with radical total abdominal hysterectomy and bilateral salpingo-oophorectomy (TAH-BSO), without lymph-node sampling, and adjuvant endocavitary brachytherapy delivering $30 \mathrm{~Gy}$ in 5 fractions in consecutive days. Treatment tolerance was excellent, with only G1 genitourinary acute toxicity and no other adverse events reported. In this experience, the choice of brachytherapy alone resulted in excellent local control outcomes. However, the patient developed a distant recurrence after 43 months and chemotherapy was applied. More research is required to determine the most effective treatment for this rare histological subtype, and in particular, the role of chemotherapy and radiotherapy combinations.

Key words: brachytherapy, endometrium, gynecology, transitional cell carcinoma.

\section{Purpose}

Transitional cell carcinomas (TCC) are rare tumors of the female genital tract, which occur more commonly in the ovary, where they account for about $2 \%$ of cancers [1]. Endometrium is a very rare site of presentation of TCC and few cases of primitive endometrial disease are reported in the literature $[2,3,4,5,6,7,8,9,10]$ (Table 1 ). Thus, it is difficult to make any recommendations relative to post-surgical adjuvant therapy in these patients, also because different adjuvant treatments, including external beam radiotherapy (EBRT), brachytherapy (BRT), chemotherapy, or observation, were used in previous cases. We report our experience with a very rare pure form of endometrial TCC treated with surgery, followed by endocavitary vaginal brachytherapy (VBT).

\section{Case presentation}

A 65-year-old woman presented with post-menopausal bleeding, occurring for at least three months. The patient underwent a hysteroscopy, which showed a small polypoid lesion located in the body of the uterus, distant from the cervix. The biopsy indicated the presence of a primitive TCC of endometrium. A contrast enhanced diagnostic abdominal-pelvic computed tomography (CT) was performed, showing a slight dilation of the uterus, with no other lesions. The patient was obese, advanced in age, on anti-hypertensive medication, with good performance status (PS 0).

A radical total abdominal hysterectomy and bilateral salpingo-oophorectomy (TAH-BSO), without lymphnode sampling was performed. Histological examination confirmed that the lesion was a rare pure form of primitive TCC of the endometrium. Macroscopically, the uterus was $70 \times 30 \times 15 \mathrm{~mm}$, enlarged by a greyish, polypoid, soft tissue mass measuring $22 \times 10 \mathrm{~mm}$. Histological examination showed that the tumor invaded less than one half of the myometrium, without diffusion to the endocervix (pT1A, pNx, according to FIGO 2009). Immunohistochemistry was positive for MNF116, CK7, and for estrogen receptors, while negative for vimentin, chromogranin, synaptophysin, CK 20, and HCG. Following surgery, the patient underwent a PET CT with 18F-fluorodeoxyglucose (18-FDG), which did not detect any suspicious uptake of the tracer.

In order to reduce the risk of vaginal recurrence, adjuvant high-dose-rate intracavitary brachytherapy 
Table 1. Previous cases of endometrial transitional cell carcinoma

\begin{tabular}{|c|c|c|c|c|}
\hline Author & No. of cases & Treatment & Follow-up & Outcomes \\
\hline Chen et al. [2] & 1 & Whole pelvic adj EBRT & $5 \mathrm{yr}$ & No evidence of recurrence \\
\hline Spiegel et al. [3] & 1 & Whole pelvic adj EBRT & $15 \mathrm{mo}$ & No evidence of recurrence \\
\hline Lininger et al. [4] & $\begin{array}{l}8 \text { (5 available for } \\
\quad \text { follow-up) }\end{array}$ & $\begin{array}{c}\text { Adj EBRT (3 pts) } \\
\text { Adj endovaginal intersti- } \\
\text { tial BRT (1 pt) } \\
\text { Adj CT (1 pt) }\end{array}$ & $3 \mathrm{mo}-12.9 \mathrm{yr}$ & $\begin{array}{l}1 \text { recurrence after } 1 \text { year from } \\
\text { adjuvant RT, } 1 \text { death by other causes }\end{array}$ \\
\hline Fukunaga et al. [5] & 1 & Adj CT & $7.6 \mathrm{yr}$ & No evidence of recurrence \\
\hline Labontè et al. [6] & 1 & $\begin{array}{c}\text { Adj EBRT + endovaginal } \\
\text { interstitial BRT }\end{array}$ & $6 \mathrm{mo}$ & No evidence of recurrence \\
\hline Mariño-Enriquez et al. [7] & 5 & No adj treatment & $3-16 \mathrm{mo}$ & No evidence of recurrence \\
\hline Lum et al. [10] & 1 & Palliative EBRT & $18 \mathrm{mo}$ & No evidence of recurrence \\
\hline Ahluwalia et al. [8] & 1 & No adj treatment & $10 \mathrm{mo}$ & No evidence of recurrence \\
\hline Ribeiro-Silva et al. [9] & 1 & No adj treatment & $1 \mathrm{yr}$ & Death by disease \\
\hline Our experience & 1 & Adj endovaginal BRT & $43 \mathrm{mo}$ & Distant progression after $43 \mathrm{mo}$ \\
\hline
\end{tabular}

EBRT - external beam radiation therapy; $C T$ - chemotherapy; BRT - brachytherapy; adj-adjuvant; mo - months; yr-years

(HDR BRT) was delivered with a Microselectron HDR device (Nucletron, Veenendaal, The Netherlands). Oncentra Brachy (Elekta, Veenendaal, The Netherlands) was used for treatment planning. The CT simulation was performed with a full bladder and empty rectum, using a slice thickness of $2.5 \mathrm{~mm}$. The proximal $3.5 \mathrm{~cm}$ of the vagina were treated. A total dose of 3,000 cGy in 5 fractions, delivered on five consecutive days was prescribed to $0.5 \mathrm{~cm}$ from the surface of the vaginal applicator.

Clinical and instrumental evaluations were scheduled every 3 months, till two years after ending of treatment. Gastrointestinal (GI) and genitourinary (GU) toxicity were evaluated using the RTOG scale. Only G1 GU dysuria was recorded as acute side effects; no GU and GI late toxicity was observed. After 43 months from the end of treatment, an abdominal/pelvic CT with intravenous contrast showed the evidence of peritoneal carcinosis; therefore, the patient started chemotherapy with carboplatin.

\section{Discussion}

We report a case of a pure form of primitive TCC of the endometrium treated with TAH-BSO and adjuvant HDR BRT, showing that this approach results in a positive outcome in terms of local control and treatment toxicity. Very few data are currently available in literature; thus, a standardized approach to the management of this disease is still an issue.

Two case reports in the 1990s were the first to present the outcomes of patients with endometrial TCC with involvement of adnexa. Both were treated with whole pelvic EBRT, and there was no evidence of disease 15 months and 5 years later, respectively [2,3], suggesting that EBRT may be a reasonable option in order to eradicate residual cancer cells in the pelvis.

Lininger et al. [4] reported outcomes of five cases of endometrial TCC, treated with surgery and various ad- juvant approaches, including EBRT, BRT (with vaginal iridium implants), and chemotherapy. With a follow-up ranging from 3 months to 12.9 years, one recurrence occurred in the vaginal cuff 1 year after radiotherapy treatment.

The potential role of chemotherapy was also investigated by Fukunaga et al. [5] in a case of a 50-year-old woman with endometrial TCC, who underwent adjuvant cyclophosphamide, cisplatin, and adryamicin, observing no evidence of disease after 7 years.

A combined adjuvant approach, pelvic EBRT, and endovaginal interstitial brachytherapy was used by Labontè et al. [6] in a patient with simultaneous Brenner tumor in the ovary and endometrial TCC. Six months after the treatment, the patient was alive without evidence of disease.

Observation was investigated in a case series of 5 patients with endometrial TCC, reporting no evidence of disease after 3-16 months [7]. Similar findings were reported by Ahluwalia et al. [8] in a patient with minimal myometrial invasion treated only with hysterectomy, while in a case report by Ribeiro-Silva et al. [9], the patient died by disease progression after one year of follow-up.

Also, the use of high-dose palliative EBRT was explored for TCC endometrial carcinoma, reporting 18 months-free of evidence of local recurrence. However, no information about the dose and fractionation schedule employed were provided [10].

Compared to literature data, our case reports a more mature follow-up, which allows to better understand the real effectiveness of adjuvant treatment. In our experience, the patient developed distant recurrence with peritoneal carcinosis after 43 months from the end of VBT, and subsequently started chemotherapy with carboplatin. As the NCCN guidelines [11] recommend, for aggressive subtypes (clear cell, carcinosarcoma or serous carcinoma) stage IA disease, the preferential approach is represented by chemotherapy and vaginal brachyther- 
apy. Unfortunately, due to the rarity of transitional cell histology, currently no data are available; nevertheless, the role of adjuvant chemotherapy and VBT may be explored in future. However, our case report supports vaginal brachytherapy as an effective adjuvant treatment, provided excellent local control with minimal toxicity.

\section{Disclosure}

The following research was conducted at the Radiotherapy Unit of the ARNAS-Civico Hospital of Palermo, Italy.

Authors report no conflict of interest.

\section{References}

1. De Cecio R, Cantile M, Collina F et al. Borderline Brenner tumor of the ovary: a case report with immunohistochemical and molecular study. J Ovar Res 2014; 7: 101.

2. Chen KT. Extraovarian transitional cell carcinoma of female genital tract. Am J Clin Pathol 1990; 94: 670-671.

3. Spiegel GW, Austin RM, Gelven PL. Transitional cell carcinoma of the endometrium. Gynecol Onc 1996; 60: 325-330.

4. Lininger RA, Ashfaq R, Albores-Saavedra J et al. Transitional cell carcinoma of the endometrium and endometrial carcinoma with transitional cell differentiation. Cancer 1997; 79: 1933-1943.

5. Fukunaga M, Ushigome S. Transitional cell carcinoma of the endometrium. Histopathology 1998; 32: 284-286.

6. Labonté S, Têtu B, Boucher D et al. Transitional cell carcinoma of the endometrium associated with a benign ovarian Brenner tumor: a case report. Hum Pathol 2001; 32: 230-232.

7. Mariño-Enríquez A, González-Rocha T, Burgos E et al. Transitional cell carcinoma of the endometrium and endometrial carcinoma with transitional cell differentiation: a clinicopathologic study of 5 cases and review of the literature. Hum Pathol 2008; 39: 1606-1613.

8. Ahluwalia M, Light AM, Surampudi K et al. Transitional cell carcinoma of the endometrium: a case report and review of the literature. Int J Gynecol Pathol 2006; 25: 378-382.

9. Ribeiro-Silva A. Immunohistochemical features of a papillary squamous cell carcinoma of the endometrium with transitional cell differentiation. Diagn Pathol 2007; 2: 26.

10. Lum D. Primary transitional cell carcinoma of the endometrium. Pathology 2005; 7: 554-557.

11. Koh WJ, Abu-Rustum NR, Bean $S$ et al. Uterine Neoplasms, Version 1.2018, NCCN Clinical Practice Guidelines in Oncology. J Natl Compr Canc Netw 2018; 16: 170-199. 